besten ein Bleizählrohr von 0,1 mm Wandstärke, außen mit Aluminium umgeben, dessen Empfindlichkeit nur in engen Grenzen schwankt. Bei sehr schwachen $\gamma$-Strahlungen von weniger als $1 \mathrm{eMV}$ Energie kann man mit Hilfe einer einfachen Relativmessung mit einem Blei- und einem Aluminiumzählrohr die ungefähre Energie der $\gamma$-Strahlung bestimmen.

c) Die Kenntnis der absoluten Nachweiswahrscheinlichkeit macht viele Anwendungen möglich. Z. B. läßt sich bei Positronenstrahlern einfach entscheiden, ob außer der Vernichtungsstrahlung noch $\gamma$-Strahlung ausgesandt wird. Bei $\beta$-Umwandlung mit $\gamma$-Strahlung läßt sich entscheiden, ob die $\gamma$-Strahlung die erwartete Häufigkeit hat, oder ob es notwendig ist, mehrfache Übergänge oder andere Prozesse anzunehmen. Bei Koinzidenzmessungen zwischen $\beta$ - und $\gamma$-Strahlen besteht jetzt die Möglichkeit, aus der Koinzidenzhäufigkeit pro $\beta$-Ausschlag die mittlere $\gamma$-Energie pro $\beta$-Strahl zu ermitteln. Man braucht dazu ein $\gamma$-Zählrohr, dessen Empfindlichkeit sich stark mit der Energie ändert, also am besten ein Aluminiumzählrohr.

d) Besonders zu erwähnen sind zwei Möglichkeiten, die sich aus den Empfindlichkeitskurven er- geben. Das Bleizählrohr variiert im Bereich von 150 bis $1500 \mathrm{ekV}$ nur wenig in der Empfindlichkeit. Man kann übersehen, daß die Unterschiede nur noch 10-15\% vom Mittelwert abweichen werden, wenn man die Bleischicht von $0,1 \mathrm{~mm}$ auf $0,2 \mathrm{~mm}$ erhöht. In diesem Bereich hat man dann ein Zählrohr konstanter Empfindlichkeit. Ein Zählausschlag entspricht dem Durchgang von $100 \gamma$-Quanten durch das Zählvolumen, unabhängig von der $\gamma$-Energie.

Andererseits ist die Empfindlichkeit des Messingzählrohrs im Bereich 0,2 bis $3 \mathrm{eMV}$ bis auf $\pm 10 \%$ proportional der $\gamma$-Energie. Man kann erwarten, daß dieser Bereich noch bis etwa 0,1 eMV ausgedehnt wird, wenn man statt 0,5 mm Messing $2 \mathrm{~mm}$ Messing verwendet. Ein solches Zählrohr mißt die durchgegangene $\gamma$-Energie. Ein Zählrohrausschlag entspricht 175 eMV durch das Zählrohrvolumen gestrahlter Energie. Damit läßt sich z. B. bei $\beta$-Strahlern bei bekannter $\beta$-Strahlstärke die $\gamma$-Energie pro Zerfall unmittelbar angeben. Durch Kombination der Messung mit Blei- und Messingzählrohr ergibt sich die mittlere Energie einer $\gamma$-Strahlung, die sonst mit Zählrohren nicht gemessen werden kann.

\title{
Die Phänomenologie zweier Verfahren zur Isotopentrennung
}

\author{
Von Alfred Klemm \\ Aus dem Kaiser-Wilhelm-Institut für Chemie, Tailfingen \\ (Z. Naturforschg. 1, 252-257 [1946]; eingegangen am 22. März 1946)
}

\begin{abstract}
1. Wenn ein Isotopengemisch von $x=0$ aus, wo die Konzentrationen konstant gehalten werden, in den positiven Halbraum hineindiffundiert, dann kann man jenseits $x$ ein Gemisch gewinnen, dessen Trennfaktor $Q$ näherungsweise durch $\ln Q=f \ln q$ gegeben ist, wo $q=D_{j} / D_{k}$ und $f=\ln \left(v^{0} / v\right)$. Dabei sind $v^{0}$ und $v$ die Mengen pro Flächeneinheit, die 0 und $x$ überschritten haben. 2. An der "wandernden Grenzfläche" stromführender Salze entsteht eine Isotopenanreicherung, wenn die Wanderungsgeschwindigkeiten $w_{j}$ und $w_{k}$ der Isotope verschieden sind. Der Trennfaktor ist näherungsweise durch $\ln Q=f \ln q$ gegeben, wo $q=w_{j} / w_{k}$ und $f=2 w^{0} t / \sqrt{\pi D t} . w^{0}$ ist die mittlere Relativgeschwindigkeit von Kat- und Anionen. Die Anreicherung erstreckt sich bis zum Abstand $\sqrt{\pi D t}$ von der Grenzfläche.
\end{abstract}

$\mathrm{B}_{\mathrm{n}}^{\mathrm{e}}$ ei den meisten Verfahren zur Isotopentrennung erhält man wegen der Ahnlichkeit des Trennfaktors $Q$ mit einer Potenz des Elementareffektes $q$ und wegen der Kleinheit von $q-1$ eine gute Näherungsformel für $Q$, wenn man in der Entwicklung von $\ln Q$ nach Potenzen von $q-1$ nur das erste Glied berücksichtigt, wenn man also schreibt

$$
\ln Q= \pm f a \text {. }
$$

Bei dieser Näherung ist es belanglos, ob $\alpha$ gleich $(q-1)$ oder $\ln q$ oder z. B. $2(\sqrt{q}-1)$ gesetzt wird. Der Elementareffekt $q$ kann die Konstante 
des Massenwirkungsgesetzes, der Quotient der Diffusionskoeffizienten oder der Quotient der Ionenwanderungsgeschwindigkeiten, $\alpha$ kann der Thermodiffusionsfaktor sein. Der Trennfaktor $Q$ für die Isotope $j$ und $k$ ist

$$
Q_{j k}=\gamma_{j} \gamma_{k}^{\prime} /\left(\gamma_{k} \gamma_{j}^{\prime}\right)
$$

$\gamma$ und $\gamma^{\prime}$ sind Molenbrüche in den zwei Gemischen, auf die sich der Trennfaktor bezieht. $f$ ist die Vervielfachung. Um Versuchsergebnisse durch Zahlenangaben für $q, \alpha$ oder $\mu$ präzis angeben zu können, ist es zweckmäßig, folgende Beziehungen zugrunde zu legen:

$$
a_{j k}=\ln q_{j k}=\mu \ln \left(m_{j} / m_{k}\right) .
$$

$m$ Isotopenmasse, $\mu$ Masseneffekt. Die Definition (3) hat den Vorteil, daß dem Reziprokwert von $q$ und $m_{j} / m_{k}$ der $\alpha$-Wert mit umgekehrtem Vorzeichen entspricht.

Gewisse Trennverfahren lassen sich in zwei Gruppen einteilen: Bei den Verfahren erster Art ist die Vervielfachung $f$ eine Funktion der Ausbeute $N$ und der umgesetzten Menge $N^{0}$; und zwar gilt ungefähr

$$
f=\ln \left(N^{0} / N\right) .
$$

Nur wenn die relative Ausbeute $N / N^{0}$ sehr klein ist, wird $f$ groß. Bei den Verfahren zweiter Art besteht kein solcher Zusammenhang zwischen $f, N$ und $N^{0}$. Man kann diese Verfahren so gestalten, daß die Vervielfachung groß und die relative Ausbeute von der Größenordnung 1 ist. Wir erläutern dies an zwei einfachen Beispielen:

a) Restverfahren. Beim Eindampfen einer Flüssigkeit werde der Dampf kontinuierlich abgeführt, aber so langsam, daß an der Grenze zwischen Flüssigkeit und Dampf stets die Gleichgewichtsverteilung gewahrt bleibt. Dann ist der Trennfaktor zwischen der Flüssigkeit und dem entweichenden Dampf

$$
q=N_{j} d N_{k} /\left(N_{k} d N_{j}\right) .
$$

$N$ Flüssigkeitsmenge, $-d N$ pro Zeitelement entwickelte Dampfmenge, $q$ Konstante des Massenwirkungsgesetzes. Die Integration ergibt

$$
N_{k}^{0} / N_{k}=\left(N_{j}^{0} / N_{j}\right)^{\mathrm{q}}
$$

$N^{0}$ ursprüngliche Flüssigkeitsmenge. Der Trennfaktor zwischen dem Rest der Flüssigkeit und der ursprünglichen Flüssigkeit ist

$$
Q=N_{j} N_{k}^{0} /\left(N_{k} N_{j}^{0}\right)=\left(N_{j}^{0} / N_{j}\right)^{q-1} .
$$

Es ist

$$
\begin{gathered}
\ln Q=f(q-1), \\
f=\ln \left(N_{j}^{0} / N_{j}\right) .
\end{gathered}
$$

Das Restverfahren ist also ein Verfahren erster Art.

b) Gegenstrom. Bei einer Rektifikationskolonne mit $z$ Kammern sei der Trennfaktor zwischen Flüssigkeit und Gas innerhalb einer Kammer gleich $q$. Ist die Kolonne im Gleichgewicht, so besitzt das in die nächste Kammer aufsteigende Gas die gleiche Isotopenzusammensetzung wie die von der nächsten Kammer herablaufende Flüssigkeit. Also ist im Gleichgewicht der Trennfaktor zwischen den Flüssigkeiten zweier aufeinander folgender Kammern $q$ und zwischen den Flüssigkeiten der ersten und letzten Kammer

$$
Q=q^{2} .
$$

Vergleicht man das gemittelte Mischungsverhältnis in der oberen Hälfte der Säule mit dem in der unteren Hälfte der Säule, so entspricht dem eine von der Zellenzahl abhängige Vervielfachung, wobei die relative Ausbeute von der Größenordnung 1 ist. Also sind Gegenstromverfahren Verfahren zweiter Art.

\section{Spitzenverfahren ${ }^{1}$}

Ein Isotopengemisch möge von der Ebene $x=0$ aus in den positiven Halbraum hineindiffundieren. Es sei dafür gesorgt, daß die Konzentrationen bei $x=0$ konstant bleiben. Dann gilt im Idealfall für jede Isotopenart $i$ die Anfangsbedingung

$$
n_{i}=0 \text { für } t=0 \text { und } 0<x<\infty,
$$

die Randbedingung

$$
n_{i}=n_{i}^{0} \text { für } x=0 \text { und } 0<t<\infty
$$

und die Differentialgleichung

$$
\frac{\partial n_{i}}{\partial t}=D_{i} \frac{\partial^{2} n_{i}}{\partial x^{2}} .
$$

$x$ Ortskoordinate, $t$ Zeit, $n$ Menge pro Volumeneinheit, $D$ Diffusionskonstante. Die Lösung lautet

$$
\begin{gathered}
n_{i}=n_{i}^{0}\left[1-\Phi\left(\zeta_{i}\right)\right], \\
\zeta_{i}=\frac{x}{2 \sqrt{D_{i} t}},
\end{gathered}
$$

$$
\Phi(\zeta)=\frac{2}{\sqrt{\pi}} \int_{0}^{\zeta} e^{-\zeta^{2}} d \zeta .
$$


Für die Menge pro Flächeneinheit

$$
v_{i}=\int_{x}^{\infty} n_{i} d x, \quad v_{i}^{0}=\int_{0}^{\infty} n_{i} d x
$$

erhält man

$$
\begin{aligned}
v_{i} & =n_{i}^{0} \frac{2}{\sqrt{\pi}} \sqrt{D_{i} t} \psi\left(\zeta_{i}\right), \\
v_{i}^{0} & =n_{i}^{0} \frac{2}{\sqrt{\pi}} \sqrt{D_{i} t}, \\
\psi(\zeta) & =e^{-\zeta^{2}}-\sqrt{\pi} \zeta[1-\Phi(\zeta)] .
\end{aligned}
$$

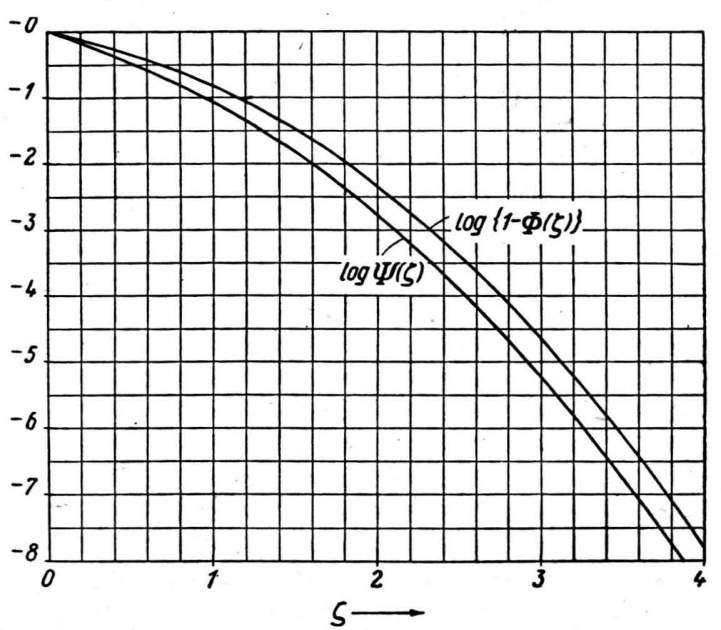

Abb. 1. Zur relativen Ausbeute beim Spitzenverfahren.

Abb. 1 zeigt die Zehnerlogarithmen der Funktionen $[1-\Phi(\zeta)]$ und $\psi(\zeta)$. Wir definieren zwei Arten von Trennfaktoren durch

$$
Q_{j k}=\frac{n_{j} n_{k}^{0}}{n_{k} n_{j}^{0}}, \quad Q_{j k}^{*}=\frac{v_{j} n_{k}^{0}}{v_{k} n_{j}^{0}} .
$$

Einsetzen von (14) und (19) in (22) und (23) ergibt

$$
\begin{aligned}
& \ln Q_{j k}=\ln \left[1-\Phi\left(\zeta_{j}\right)\right]-\ln \left[1-\Phi\left(\zeta_{k}\right)\right], \\
& \ln Q_{j k}^{*}=\frac{1}{2} \ln D_{j} / D_{k}+\ln \psi\left(\zeta_{j}\right)-\ln \psi\left(\zeta_{k}\right) .
\end{aligned}
$$

Für $D_{j} / D_{k} \rightarrow 1$ wird aus (24) und(25) näherungsweise

$$
\begin{aligned}
& \ln Q_{j k}=\frac{\partial \ln [1-\Phi]}{\partial \zeta} \frac{\partial \zeta}{\partial D}\left(D_{j}-D_{k}\right), \\
& \ln Q_{j k}^{*}=\left(\frac{1}{2 D}+\frac{\partial \ln \psi}{\partial \zeta} \frac{\partial \zeta}{\partial D}\right)\left(D_{j}-D_{k}\right) .
\end{aligned}
$$

Einsetzen von (15), (16) und (21) in (26) und (27) ergibt

$$
\begin{aligned}
\ln Q_{j k}=f a_{j k}, \quad f & =\frac{\zeta \exp \left(-\zeta^{2}\right)}{\sqrt{\pi}[1-\Phi(\zeta)]}, \\
\ln Q_{j k}^{*}=t^{*} a_{j k}, f^{*} & =\frac{\exp \left(-\zeta^{2}\right)}{2 \psi(\zeta)}, \\
a_{j k} & =\left(D_{j}-D_{k}\right) / D .
\end{aligned}
$$

Der Elementareffekt ist

$$
q_{j k}=D_{j} / D_{k} .
$$

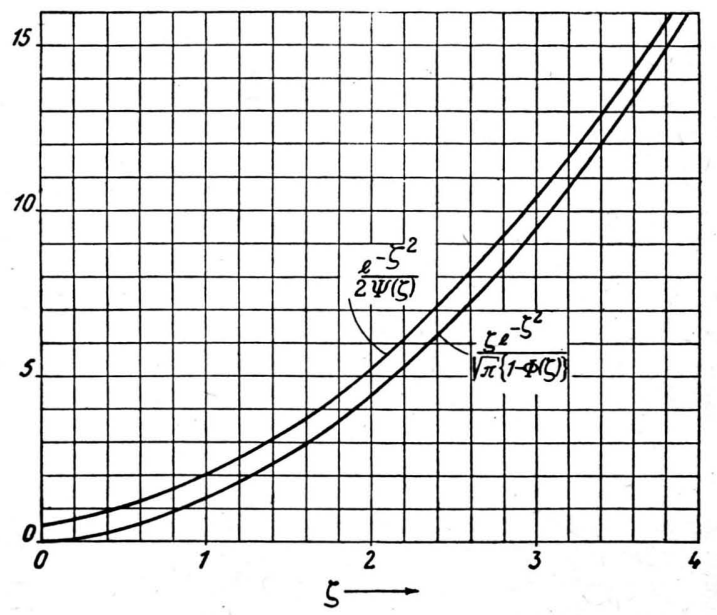

Abb. 2. Die Vervielfachung beim Spitzenverfahren.

Die Vervielfältigung $f(\zeta)$ bzw. $f^{*}(\zeta)$ gemäß (29) bzw. (31) ist in Abb. 2 dargestellt. Für $\zeta \rightarrow \infty$ gilt

$$
\begin{aligned}
1-\Phi(\zeta) & =\frac{\exp \left(-\zeta^{2}\right)}{\sqrt{\pi} \zeta}, \\
\psi(\zeta) & =\frac{\exp \left(-\zeta^{2}\right)}{2 \zeta^{2}} .
\end{aligned}
$$

Einsetzen von (34) und (35) in (19), (29) und (31) ergibt die für $\zeta \rightarrow \infty$ gültigen Beziehungen

$$
\ln \left(\nu^{0} / v\right)=f=f^{*}=\zeta^{2} .
$$

Die relative Ausbeute $v / v$ ist also logarithmiert für $q_{j k} \rightarrow 1$ und $\zeta \rightarrow \infty$ gleich der Vervielfachung, d. h. das Spitzenverfahren ist den Verfahren erster Art zuzuordnen. In Tab. 1 sind für $\sqrt{q}=1,1$ und $\alpha=0,2$ Werte für $Q$ und $Q^{*}$ eingetragen, die nach (24) und (25) bzw. (28) und (30) ausgerechnet worden sind.

Als Beispiel schildern wir einen Versuch, über den schon früher berichtet wurde ${ }^{1}$. Es wurde eine Säule von $5 \mathrm{~cm}$ Länge und 2,5 cm Durchmesser her- 


$\begin{array}{ccccc}\zeta_{j} & \begin{array}{c}\mathrm{Q} \\ \text { exakt }\end{array} & \begin{array}{c}\mathrm{Q} \\ \text { Näherung }\end{array} & \begin{array}{c}\mathrm{Q}^{*} \\ \text { exakt }\end{array} & \begin{array}{c}\mathrm{Q}^{*} \\ \text { Näherung }\end{array} \\ 0 & 1 & 1 & 1,1 & 1,105 \\ 1 & 1,31 & 1,3 & 1,52 & 1,51 \\ 2 & 2,5 & 2,4 & 3,0 & 2,9 \\ 3 & 7,2 & 6,6 & 8,6 & 7,9 \\ 4 & 32 & 27 & 38 & 33\end{array}$

Tab. 1.

gestellt, die auf der einen Seite $(1 \mathrm{~cm})$ aus $\mathrm{Cu}_{2} \mathrm{~S}$ und auf der anderen Seite $(4 \mathrm{~cm})$ aus $\mathrm{Ag}_{2} \mathrm{~S}$ bestand. Diese Säule wurde während 12 Stdn. auf $450^{\circ} \mathrm{C}$ gehalten. Die $\mathrm{Cu}$ - und $\mathrm{Ag}$-Ionen sind in den genannten Salzen bei Zimmertemperatur praktisch unbeweglich, oberhalb des Umwandlungspunktes $\left(91^{\circ} \mathrm{C}\right.$ bei $\mathrm{Cu}_{2} \mathrm{~S}, 179^{\circ} \mathrm{C}$ bei $\left.\mathrm{Ag}_{2} \mathrm{~S}\right)$ dagegen sehr beweglich. Während der 12 Stdn. trat also eine unvollständige Vermischung der $\mathrm{Cu}$ - und $\mathrm{Ag}$-Ionen ein. Nach Abkühlung wurde am Ende des $\mathrm{Ag}_{2} \mathrm{~S}$ Teiles ein $1,5 \mathrm{~cm}$ dickes Stück abgeschnitten und daraus wurden einige $10 \mathrm{mg} \mathrm{Cu}$ gewonnen. Die massenspektrographische Analyse ergab als Trennfaktor dieses Kupfers in bezug auf natürliches Kupfer ungefähr 1,055. Wenn die Isotopenhäufigkeiten beim natürlichen Kupfer $70,1 \%{ }^{63} \mathrm{Cu}$ und ${ }^{29,9 \%}{ }^{65} \mathrm{Cu}$ sind ${ }^{2}$, so enthielt das angereicherte Kupfer ungefähr $71,1 \%{ }^{63} \mathrm{Cu}$ und $28,8 \%{ }^{65} \mathrm{Cu}$. Die Theorie für den Halbraum ist bei der gewählten Verkürzung der Anordnung im Vergleich zur Meßgenauigkeit ohne beachtlichen Fehler anwendbar. Die ursprünglich scharfe Grenze zwischen $\mathrm{Cu}_{2} \mathrm{~S}$ und $\mathrm{Ag}_{2} \mathrm{~S}$ ist die Ebene $x=0$. Dort herrscht die zeitlich konstante $\mathrm{Cu}-\mathrm{Konzentration} \boldsymbol{n}^{0}$, die gleich der halben $\mathrm{Cu}-\mathrm{Konzentration}$ im reinen $\mathrm{Cu}_{2} \mathrm{~S}$ ist. Die in der Theorie vorkommenden Größen waren beim Experiment:

$D=1,1 \mathrm{~cm}^{2} / \mathrm{d}, n^{0}=2,25 \mathrm{~g} \mathrm{Cu} / \mathrm{cm}^{3}, \ln \left(m_{2} / m_{1}\right)$ $=0,031, x=2,5 \mathrm{~cm}, t=0,5 \mathrm{~d}$. Daraus folgt: $\zeta=$ $1,68, \psi(\zeta)=7,8 \cdot 10^{-3}, \nu=14,6 \mathrm{mg} \mathrm{Cu} / \mathrm{cm}^{2}, f^{*}=4$. Massenspektrographisch wurde gefunden: $\ln Q^{*}$ $=0,055 \pm 0,025$. Daraus folgt: $\ln \left(D_{1} / D_{2}\right)=0,0137$ $\pm 0,0062, \mu=-0,44 \pm 0,2$.

\section{Stauverfahren ${ }^{3}$}

Zur Beschreibung des Stauverfahrens wollen wir uns einen konkreten Fall vorstellen: Der positive Halbraum sei mit geschmolzenem $\mathrm{PbCl}_{2}$ erfüllt, der negative mit geschmolzenem TlCl. Es werde nun

2 H. Ew a l'd, Z. Physik 122, 487 [1944].

3 A. K l e m m, Naturwiss. 32, 69 [1944]; Z. Physik 123,10 [1944]. ein elektrisches Feld in der $x$-Richtung angelegt. Dann werden die Kationen im Mittel relativ zu den Chlorionen sehr bald eine stationäre ortsunabhängige Wanderungsgeschwindigkeit $w^{0}$ annehmen. Das ist trotz der größeren Leitfähigkeit von $\mathrm{PbCl}_{2}$ gegenüber dem $\mathrm{TlCl}$ dadurch möglich, daß sich an der Grenzfläche infolge Abwanderns der $\mathrm{Pb}$-Ionen ein Chlorionenüberschuß und damit eine negative Raumladung bildet, deren Feld sich dem äußeren Feld überlagert. Die dabei entstehenden Konzentrationsänderungen sind vernachlässigbar klein. Die Grenzfläche bleibt bis auf eine geringfügige Diffusionsverbreiterung scharf, weil sich $\mathrm{Pb}$ - und Tl-Ionen, die an Stellen gleicher Feldstärke sind, wegen des Unterschiedes ihrer Beweglichkeiten im Sinne einer Verschärfung der Grenzfläche gegeneinander verschieben. Die Ebene $x=0$ sei für alle Zeiten die Grenzfläche.

Die $z$ Bleiisotope mögen nun die Konzentrationen $n_{i}$, die ortsunabhängigen Anfangskonzentrationen $n_{i}^{0}$, die konstanten Diffusionskoeffizienten $D_{i}$ und an Stellen ohne Konzentrationsgradienten die Wanderungsgeschwindigkeiten $w_{i}$ relativ $\mathrm{zu}$ den Chlorionen besitzen. Die $(z-1)$ Elementareffekte

$$
q_{j k}=w_{j} / w_{k}
$$

seien konstant. Die $w_{i}$ sind wegen der durch Raumladungen entstehenden Ortsabhängigkeit der Feldstärke Funktionen von $x$ und $t$. Die Summe der Teilchenströme ist null:

$$
\sum_{i=1}^{i=z}\left[\left(w_{i}-w^{0}\right) n_{i}-D_{i} \frac{\partial n_{i}}{\partial x_{i}^{i}}\right]=0 .
$$

Es gelten die $z$ Differentialgleichungen

$\frac{\partial n_{i}}{\partial t}=-\left(w_{i}-w^{0}\right) \frac{\partial n_{i}}{\partial x}-n_{i} \frac{\partial w_{i}}{\partial x}+D_{i} \frac{\partial^{2} n_{i}}{\partial x^{2}}$.

Die $z$ Anfangsbedingungen lauten

$$
n_{i}=n_{i}^{0} \text { für } t=0 \text { und } 0<x<\infty,
$$

und die $z$ Randbedingungen

$\left(w_{i}-w^{0}\right) n_{i}-D_{i} \frac{\partial n_{i}^{\prime}}{\partial x_{i}}=0$ für $x=0$ und $0<t<\infty$.

Da die vollständige Integration dieses Gleichungssystems zu schwierig ist, machen wir die Annahme, daß außer einem häufigen Isotop nur Isotope beteiligt sind, deren Konzentrationen zu jeder Zeit sehr klein sind. Dann ist die Leitfähigkeit und deshalb die Feldstärke und deshalb $w_{i}$ 
konstant, und wir haben $z$ unabhängige Differentialgleichungen

$$
\frac{\partial n_{i}}{\partial t}=-\left(w_{i}-u^{0}\right) \frac{\partial n_{i}}{\partial x}+D_{i} \frac{\partial^{2} n_{i}}{\partial x^{2}}
$$

und $z$ unabhängige Randbedingungen (41). Die Lösung kann man einer Arbeit von M. M a s o n und W. We ave $\mathrm{r}^{4}$ entnehmen. Sie lautet

$$
\begin{gathered}
n_{i}=n_{i}^{0} Q\left(\zeta_{i}, \vartheta_{i}\right) \\
\Omega(\zeta, \vartheta)=-\frac{2}{\sqrt{\pi}} \vartheta e^{-(\vartheta-\zeta)^{2}}+\frac{1}{2}[1-\Phi(\vartheta-\zeta)] \\
+\frac{1}{2}[1+4 \vartheta(\vartheta+\zeta)][1-\Phi(\vartheta+\zeta)] e^{4 \zeta \vartheta} \\
\zeta_{i}=\frac{x}{2 \sqrt{D_{i} t}}, \vartheta_{i}=\frac{w_{i}-w^{0}}{2} \sqrt{\frac{t}{D_{i}}}
\end{gathered}
$$

Ist $j$ eines der seltenen und $k$ das häufige Isotop, so ist der Trennfaktor

gegeben durch

$$
Q_{j k}=n_{j} n_{k}^{0} /\left(n_{k} n_{j}^{0}\right)
$$

und es ist

$$
Q_{j k}=Q\left(\zeta_{j}, \vartheta_{j}\right)
$$

$$
w_{j}-w^{0}=w_{j}-w_{k} .
$$

Ist $j$ das häufige und $k$ eines der seltenen Isotope, so hat man

und

$$
Q_{j k}=1 / \Omega\left(\zeta_{k}, \vartheta_{k}\right)
$$

$$
w_{k}-w^{0}=-\left(w_{j}-w_{k}\right) .
$$

Allgemein gilt

$$
\ln Q_{j k}=\ln \Omega\left(\zeta_{j}, \vartheta_{j}\right)-\ln \Omega\left(\zeta_{k}, \vartheta_{k}\right),
$$

und näherungsweise für

$$
D_{j} / D_{k} \rightarrow 1 \text { und } w_{j} / u_{k} \rightarrow 1:
$$

$$
\begin{aligned}
& \ln Q_{j k}=\frac{\partial \ln \Omega}{\partial \zeta} \frac{\partial \zeta}{\partial D}\left(D_{j}-D_{k}\right) \\
& +\frac{\partial \ln Q}{\partial \vartheta}\left[\frac{\partial \vartheta}{\partial D}\left(D_{j}-D_{k}\right)+\frac{\partial \vartheta}{\partial w}\left(w_{j}-w_{k}\right)\right] .
\end{aligned}
$$

Da in (53) $\partial \vartheta / \partial D$ sowie $\partial \vartheta / \partial w$ für $w_{j}=w_{k}=w^{0}$ und $\partial \ln \Omega / \partial \zeta$ sowie $\partial \ln \Omega / \partial \vartheta$ für $\vartheta=0$ zubilden ist, reduziert sich (53) $\mathrm{zu}$

$$
\ln Q_{j k}=\left(\frac{\partial \Omega}{\partial \vartheta}\right)_{\vartheta=0} \frac{1}{2} \sqrt{\frac{t}{D}}\left(w_{j}-w_{k}\right) .
$$

4 Physic. Rev. 23, 424 [1924].
Durch Einsetzen von (44) in (54) ergibt sich

$$
\begin{aligned}
\ln Q_{j k} & =-f a_{j k}, \\
f & =\left(2 w^{0} t / \sqrt{\pi D t}\right) \psi(\zeta), \\
a_{j k} & =\left(w_{j}-w_{k}\right) / w^{0} .
\end{aligned}
$$

$\psi(\zeta)$ ist durch (21) definiert. In Abb. 3 ist $Q_{j k}$ für $x=0$ als Funktion von $1 / 2\left(w_{j}-w_{k}\right) \sqrt{t / D}$ nach

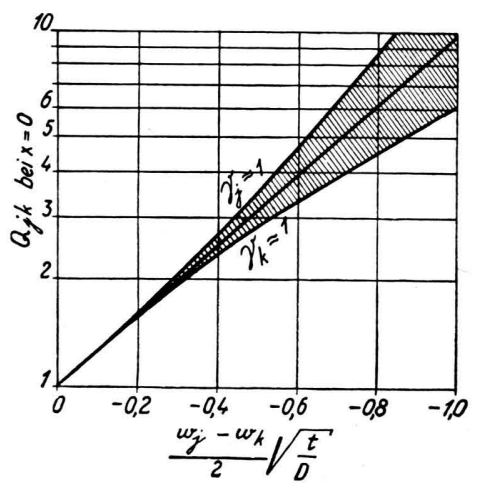

Abb. 3. Der Trennfaktor an der stauenden Grenzfläche.

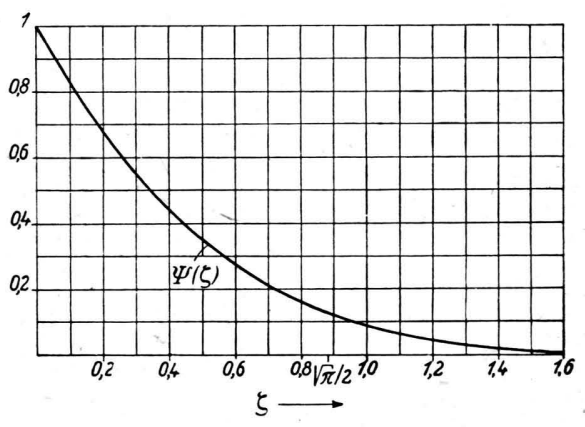

Abb. 4. Der räumliche Verlauf des Trennfaktors beim Stauverfahren.

(48), (50) und (55) eingetragen. In dem schraffierten Gebiet der Abb. 3 müssen alle $Q_{j k}$ für $x=0$ und beliebige Isotopenmischungen $\left(w_{i}==\right.$ konstant) liegen. Die Näherung (55) ist am Anfang des Stauvorganges für beliebige Mischungen brauchbar und zeigt, daß das Stauverfahren ein Verfahren zweiter Art ist.

In Abb. 4 ist $\psi(\zeta)$ nach (21) als Funktion von $\zeta$ aufgetragen. $\psi(\zeta)$ läßt sich schematisieren durch eine Funktion $\psi^{\prime}(\zeta)$, die definiert ist durch

$$
\left.\begin{array}{ll}
\psi^{\prime}(\zeta)=1-2 \zeta / \sqrt{\pi} & \text { für } 0<\zeta<\sqrt{\pi} / 2, \\
\psi^{\prime}(\zeta)=0 & \text { für } \sqrt{\pi} / 2<\zeta<\infty .
\end{array}\right\}
$$


$\psi(\zeta)$ ähnelt $\psi^{\prime}(\zeta)$, denn die Ableitungen beider Funktionen sind $\leqslant 0, \psi(0)=\psi^{\prime}(0)=1$ und

$$
\int_{0}^{\infty} \psi(\zeta) d \zeta=\int_{0}^{\infty} \psi^{\prime}(\zeta) d \zeta=\frac{\sqrt{\pi}}{4} .
$$

Die praktische Reichweite der Anreicherung ist derjenige Abstand $x$ von der Grenzfläche, bei dem $\psi^{\prime}(\zeta)$ verschwindet, also

$$
x=\sqrt{\pi D} \bar{t} .
$$

Die Näherungslösung (55) erhält man auch, wenn man (39) und (41) in für den Anfang des Stauvorganges zulässiger Weise ändert. Man schreibe nämlich (39) und (41) in der Form

$$
\begin{array}{r}
\frac{\partial \ln n_{i}}{\partial t}=-\left(w_{i}-w^{0}\right) \frac{\partial \ln n_{i}}{\partial x}-\frac{\partial u_{i}}{\partial x} \\
+D_{i}\left(\frac{\partial \ln n_{i}}{\partial}\right)^{2}+D_{i} \frac{\partial^{2} \ln n_{i}}{\partial x^{2}}, \\
\left(w_{i}-w^{0}\right)-D_{i} \frac{\partial \ln n_{i}}{\partial x}=0 \text { bei } x=0,
\end{array}
$$

setze $w_{i}=$ konstant und streiche in (61) die Glieder mit $\partial \ln n_{i} / \partial x$, weil aus (62) zusammen mit der Anfangsbedingung (40) folgt, daß $\partial^{2} \ln n_{\iota} / \partial x^{2}$ für $t=0$ bei $x=0$ unendlich groß ist. Man hat dann statt $(61)$

$$
\frac{\partial \ln n_{i}}{\partial t}=D_{i} \frac{\partial^{2} \ln n_{i}}{\partial x^{2}} .
$$

Diẹ Lösung von (63) für (62) und (40) lautet

$$
\ln \left(n_{i} / n_{i}^{0}\right)=-(4 / \sqrt{\pi}) \vartheta_{i} \psi\left(\zeta_{i}\right) .
$$

Sie führt, in (53) statt $\ln \Omega\left(\zeta_{i}, \vartheta_{i}\right)$ eingesetzt, zu (55).
Zum Schluß bringen wir eine Utberlegung, die bei der-praktischen Bestimmung des Elementareffektes nützlich sein kann: Wir denken uns einen mit $\mathrm{PbCl}_{2}$ gefüllten Behälter mit den Molinhalten $N_{i}$, der zwei Öffnungen für den Ein- und Austritt des Stromes besitzt. In Öffnung 1 befinde sich die stauende Grenzfläche. Öffnung 2(Querschnitt $S$ ) möge genügend weit von Öffnung 1 entfernt sein, so daß die Konzentrationen $n_{i}$ in und in der Umgebung der Öffnung 2 während der Versuchsdauer konstant bleiben. Dann ist ein Molinhalt des Behälters nach der Zeit $t$ :

$$
N_{i}=N_{i}^{0}-S n_{i}^{0} \frac{w_{i}-w^{0}}{w^{0}} \int_{0}^{t} w^{0} d t .
$$

Nun ist die durch Öffnung 2 getretene Ladungsmenge $Q$ :

$$
Q=S F n_{\mathrm{Cl}}^{0} \int_{0}^{t} w^{0} d t .
$$

F Faraday-Konstante. Einsetzen von (66) in (65) ergibt

$$
\frac{w_{i}-w^{0}}{u^{0}}=\frac{N_{i}^{0}-N_{i}}{N_{i}^{0}} \frac{F N_{\mathrm{Cl}}^{0}}{Q},
$$

und für zwei Isotope $j$ und $k$ :

$$
\frac{w_{j}-w_{k}}{w^{0}}=-\left(\frac{N_{j}}{N_{j}^{0}}-\frac{N_{k}}{N_{k}^{0}}\right) \frac{F N_{\mathrm{C} 1}^{0}}{Q} .
$$

Die Benützung von (68) zur Bestimmung des Elementareffektes hat den Vorteil, daß man den Diffusionskoeffizienten nicht zu kennen braucht und daß Konvektion im Behälter zulässig ist, solange kein angereichertes Gemisch bis zur Öffnung 2 gelangt. 